\section{Revista 2019 \\ Facultad Vol. 15(1) \\ de Ciencias Básicas enero-junio 2019}

\title{
Composición química del aceite esencial de flores De Salvia leucantha Cav. (Lamiaceae)
}

\author{
William Fernando Castrillón Cardona ${ }^{a}$, Javier Andrés Matulevich Peláeza, \\ Jefferson Alberto Rodríguez Martíneza , Diego Alejandro Silva Carreroa
}

Resumen: El aceite esencial (AE), de flores de Salvia leucantha Cav, se obtuvo mediante destilación por arrastre con vapor como método de extracción, con un rendimiento del $0.16 \%$. Para la determinación de la composición del Ae de S. leucantha Cav se utilizó un cromatógrafo de gases, acoplado a un espectrómetro de masas (GC-MS) Shimadzu QP-2010 plus; los resultados se compararon con la base de datos NITS-08 y los índices de retención (IR) reportados en la literatura. Se estableció la presencia de monoterpenos (M) como acetato de bornilo (9.8\%), a-pineno (4.4\%), delta d-canfeno (3.3\%), $\beta$-pineno (2.4\%), sesquiterpenos (s) como cariofileno (10.0\%), $\beta$-farneseno (10.7\%), germacreno D (10.6\%) y aristoleno (17.2\%). Se resalta este último como componente mayoritario presente en el Ae de las flores de S. leucantha; además, se identificaron dos hidrocarburos de cadena alifática denominados "octadecano" y "eicosano".

Palabras Clave: aceite esencial; aristoleno; flores; Salvia leucantha

Recibido: 22 de agosto de 2018

Evaluado: 7 de noviembre de 2018

Aceptado: 7 de marzo de 2019

Disponible: 2 de marzo de 2020

Cómo citar: Castrillon Cardona, W. F., Matulevich Pelaez, J. A., Rodríguez Martínez, J. A., \& Silva Carrero, D. A. (2020). Composición química del aceite esencial de flores De Salvia leucantha Cav. (Lamiaceae). Revista Facultad De Ciencias Básicas, 15(1), 41-46. https://doi.org/10.18359/ rfcb.3615

a Laboratorio de Productos Naturales Vegetales, Universidad Distrital Francisco José de Caldas, Proyecto Curricular de Licenciatura en Química, Bogotá, Colombia. 


\title{
Chemical Composition of the Essential oil Extracted from Salvia Leucantha Cav. (Lamiaceae) Flowers
}

\begin{abstract}
The essential oil (AE) in Salvia Leucantha Cav. flowers was extracted by steam distillation with a yield of $0.16 \%$. To determine the composition of the S. leucantha Cav. essential oil, the authors used a gas chromatograph coupled to a Shimadzu QP-2010 plus mass spectrometer. Next, the results were compared against the NITS-08 database and the retention rates (IR) reported in the literature. In addition, the results established the presence of monoterpenes (M) such as, bornyl acetate (9.8\%), a-pinene (4.4\%), d-camphene (3.3\%), and $\beta$-pinene (2.4\%), and of sesquiterpenes (S), such as karyophylene (10.0\%), $\beta$-farnesene (10.7\%), germacrene D (10.6\%), and aristolene (17.2\%), wherein aristolene stands out as a major component of the essential oil extracted from S. leucantha flowers. Lastly, two aliphatic chain hydrocarbons known as "octadecane" and "icosane" were further identified.
\end{abstract}

Keywords: Essential Oil; Aristolene; Flowers; Salvia leucantha

\section{Composição química do óleo essencial extraído das flores de Salvia leucantha Cav. (Lamiaceae)}

\begin{abstract}
Resumo: O óleo essencial (AE) das flores de Salvia leucantha Cav. foi extraído através de destilação por arraste de vapor, com um rendimento de 0,16\%. Para determinar a composição desse óleo essencial, utilizou-se cromatografia gasosa com um espectrômetro de massas Shimadzu QP-2010 plus acoplado. Em seguida, os resultados foram comparados com a base de dados NITS-08 e com os índices de retenção (IR) relatados na literatura. Os resultados estabeleceram a presença de monoterpenos (M), como o acetato de bornila (9,8\%), a-pineno $(4,4 \%)$, d-canfeno $(3,3 \%)$ e $^{2}$-pineno $(2,4 \%)$, e de sesquiterpenos (s), como o cariofileno $(10,0 \%){ }^{2}$-farneseno $(10,7 \%)$, germacreno $D(10,6 \%)$ e aristoleno (17.2\%), sendo que o aristoleno é o componente majoritário do óleo essencial extraído das flores de $S$. leucantha. Por último, também foram identificados dois hidrocarbonetos de cadeia alifática,o octadecano e o eicosano.
\end{abstract}

Palavras-chave: óleo essencial; aristoleno; flores; Salvia leucantha 


\section{Introducción}

Salvia es un género perteneciente a la familia de las Labiadas (Lamiaceae), con gran presencia en el continente americano (131 especies) y amplia distribución en el norte de este (Fernandez, 2006). El género Salvia es ampliamente conocido como un género de hierbas o arbustos medicinales. Se han reportado diversas actividades para sus compuestos volátiles y fijos tales como actividades citotóxicas, antimicrobianas, antiprotozoarias, antioxidante e insecticida (Jassbi et al., 2016); algunas especies de Salvia se conocen por sus actividades específicas, tal es el caso de $S$. miltiorrhiza, usada para el tratamiento de menorragia, afecciones del corazón y del hígado (Jassbi et al., 2016).

$S$. leucantha es una especie nativa de México con numerosas actividades reportadas (Amir et al., 2016). Algunas de ellas son, por ejemplo, la neurotrópica reportada por Jassbi et al. (2016), o la actividad citotóxica para sus compuestos de tipo abietano reportada por Aoyagi et al. (2008). En la actualidad, S. leucantha ha sido estudiada en partes aéreas sin diferenciación entre flores, hojas o tallos, y se han reportado cuatro nuevos diterpenos de tipo neo-clerodano (Lia et al., 2018).

La composición química del aceite esencial de la especie $S$. leucantha en partes aéreas colectada en Nainital, India, ha sido reportada por Negi et al. (2007), y se obtuvieron sesquiterpenos como b-cariofileno (13.9\%), a-guaienano (12.6\%) y germacreno-D (13.8\%), los cuales corresponden a los componentes mayoritarios. Uno de los reportes más recientes fue publicado por Ali et al. (2014) en Winslow, EE. UU., en el que determinan la composición del aceite esencial en la misma especie, lo que arroja como resultado óxido de cariofileno $(13.5 \%)$, atribuible al componente mayoritario acompañado de algunos otros como acetato de borneol (11.4\%) Y B-CARIOFILENO (6.5\%).

En Colombia, pocos estudios han sido realizados para S. leucantha. Uno de ellos lo realizaron Cely et al. (2014), quienes publicaron una primera aproximación a la composición química de la especie en el país y reportaron compuestos de tipo triterpeno y esterol. De acuerdo con lo anterior, el presente trabajo permite contribuir al conocimiento químico de S. leucantha por medio del estudio de metabolitos volátiles presentes en sus flores.

\section{Materiales y métodos}

\section{Recolección y clasificación del material vegetal}

El material vegetal (flores) se colectó en el municipio de Guavio, departamento de Cundinamarca, con coordenadas geográficas $\left(04^{\circ} 56^{\prime} 10.58^{\prime \prime} \mathrm{N}, 73^{\circ}\right.$ $50^{\prime} 5.97^{\prime \prime}$ O), a una altura de 2668 m s.n.m., en el mes de junio del 2015. Una muestra testigo se envió al Herbario Nacional de Colombia para su determinación taxonómica, donde se clasificó como Salvia leucantha cav. bajo el número de colección col 0019, identificada por el biólogo Carlos Alberto Parra.

\section{Obtención del aceite esencial y determinación de la composición química por GC-MS}

La obtención del aceite esencial de S. leucantha se dio por medio de destilación con arrastre de vapor, a partir de 511.2 g de flores frescas reducidas de tamaño y con un tiempo de extracción de tres horas; se obtuvo un líquido translucido $(0.4 \mathrm{ml})$ con un porcentaje de rendimiento del $0.16 \%$.

La determinación de la composición química del aceite esencial se realizó por GC-MS en un equipo Shimadzu QP2010 plus. La separación se realizó en una columna capilar SHRXi-5MS de $30 \mathrm{~m}$ x 0.25 $\mathrm{mm} \times 0.25 \mu \mathrm{m}$ con un modo de inyección Splitless (10:1), el gas de arrastre utilizado fue helio (grado 5.0) con flujo constante de $1.2 \mathrm{ml} / \mathrm{min}$, y la programación de la temperatura del horno fue de 40 ${ }^{\circ} \mathrm{C}$ durante cinco minutos con incrementos de $4{ }^{\circ} \mathrm{C}$ por minuto hasta $160^{\circ} \mathrm{C}$. Luego de esto se alcanzó una temperatura de $220^{\circ} \mathrm{C}$ a una velocidad de 2,5 ${ }^{\circ} \mathrm{C} /$ min y, finalmente, hasta $280^{\circ} \mathrm{C}$ a una velocidad de $8^{\circ} \mathrm{C} / \mathrm{min}$; se mantuvo a esta temperatura durante cuatro minutos, para un tiempo total de análisis de cinco minutos. Los espectros de masas se obtuvieron por ionización electrónica (ei) a $70 \mathrm{eV}$, una temperatura de línea de transferencia de $275^{\circ} \mathrm{C}$ y 
de la cámara de ionización de $230^{\circ} \mathrm{C}$. Los índices (IR) se calcularon de acuerdo con los tiempos de retención de una serie homologa de patrones de hidrocarburos desde C8 hasta C20, analizados bajo las mismas condiciones que el aceite esencial.

La identificación de los componentes presentes en el aceite esencial de la especie S. leucantha se realizó mediante la comparación de los índices de retención reportados en la literatura (Adams 2007; Goodner 2008) y los espectros de masas almacenados en la librería Nist 08. La composición porcentual de cada uno de los constituyentes del aceite se realizó de manera relativa teniendo en cuenta el porcentaje de área de cada señal en la corriente iónica total obtenida.

\section{Resultados y discusión}

El análisis de la corriente iónica total (TIC) permitió observar 30 señales, de las cuales 22 fueron identificadas, correspondientes a compuestos de características variadas para el aceite esencial extraído de flores de S. leucantha; las señales se analizaron mediante la utilización de la biblioteca NIST 08 y su comparación mediante los espectros de masas y los índices de retención reportados en la literatura, de modo que se establecieron algunos monotérpenos, hidrocarburos alifáticos y sesquiterpenos.

El análisis permitió determinar 22 compuestos, lo que corresponde al 79.6\% de la composición química total; se describieron seis monoterpenos (m), 14 sesquiterpenos (S), y dos hidrocarburos alifáticos (HA). Entre los sesquiterpenos encontrados (58.9\%) se destacan por su presencia mayoritaria dentro del aceite esencial el aristoleno (17.2\%), el germacreno-D (10.6\%), el $\beta$-farneseno con $10.7 \%$ y el cariofileno con un porcentaje de área de $10.0 \%$. Los monoterpenos representan el $20.2 \%$ y se reportan como constituyentes en mayor porcentaje el acetato de bornilo (9.8\%), el a-pineno y el $\beta$-pineno con $4.4 \%$ y $2.5 \%$, respectivamente, además del d-canfeno (3.3\%), valores que se pueden observar en la (Tabla 1).

Tabla 1. Composición química del aceite esencial obtenido en la columna SHRXi-5MS

\begin{tabular}{|c|c|c|c|c|c|c|}
\hline N. ${ }^{0}$ señal & $t_{r x}$ & IR cal. & IR Ref. & Nombre del compuesto & $\%$ & * \\
\hline 1 & 12.015 & 923.2 & 936.1 & $\alpha$-pineno & 4.4 & M \\
\hline 2 & 12.609 & 940.39 & 950.3 & $\delta$-canfeno & 3.3 & M \\
\hline 3 & 13.786 & 975.73 & 977.3 & $\beta$-pineno & 2.5 & M \\
\hline 4 & 14.473 & 996.2 & 989.2 & $\beta-\mu \iota \rho \chi \varepsilon \vee о$ & 0.2 & M \\
\hline 5 & 17.193 & 1049.63 & 1059.7 & $\gamma$-terpineno & 0.1 & M \\
\hline 6 & 22.866 & 1110.45 & 1106.3 & Octadecano & 0.1 & $\mathrm{HA}$ \\
\hline 7 & 25.944 & 1291.15 & 1285.9 & Acetato de bornilo & 9.8 & M \\
\hline 8 & 27.619 & 1296.04 & 1283.5 & $\gamma$-elemeno & 4.9 & s \\
\hline 9 & 28.799 & 1377.41 & 1376.2 & Copaeno & 0.4 & s \\
\hline 10 & 29.097 & 1387.02 & 1384.2 & $\beta$-bourboneno & 0.3 & s \\
\hline 11 & 29.339 & 1391.6 & 1390.4 & $\beta$-Elemeno & 1.0 & s \\
\hline 12 & 30.459 & 1421.15 & 1420.1 & Cariofileno & 10.0 & s \\
\hline 13 & 30.611 & 1390.14 & 1410.12 & $\beta$-cubebeno & 1.3 & s \\
\hline 14 & 31.341 & 1416.21 & 1421.4 & Aristoleno & 17.2 & S \\
\hline 15 & 31.560 & 1461.87 & 1455.9 & $\beta$-farneseno & 10.7 & $S$ \\
\hline 16 & 32.444 & 1483.73 & 1480.6 & Germacreno D & 10.6 & S \\
\hline
\end{tabular}




\begin{tabular}{|c|c|c|c|c|c|c|}
\hline N. ${ }^{0}$ señal & $t_{r x}$ & IR cal. & IR Ref. & Nombre del compuesto & $\%$ & * \\
\hline 17 & 33.237 & 1512.37 & 1498.3 & $\gamma$-muroleno & 0.3 & $\mathrm{~s}$ \\
\hline 18 & 33.466 & 1510.87 & 1523.2 & $\beta$-cadineno & 0.4 & $\mathrm{~s}$ \\
\hline 19 & 35.252 & 1570.8 & 1580.6 & Óxido de cariofileno & 1.2 & $\mathrm{~s}$ \\
\hline 20 & 36.317 & 1589.28 & 1574.2 & Germacreno D -4- ol & 0.3 & $\mathrm{~s}$ \\
\hline 21 & 47.825 & 1591.12 & 1610.1 & Isocariofileno & 0.2 & $\mathrm{~s}$ \\
\hline 22 & 58.547 & 2033.18 & 2018 & Eicosano & 0.4 & HA \\
\hline
\end{tabular}

tR: Tiempo de retención columna SHRXi-5MS; IR cal: Índice de retención calculado; IR Ref: Índice de retención de referencia (Adams, R. 2007) \%: Porcentaje relativo en el aceite esencial; * Monoterpenos (M), sesquiterpenos (S), Hidrocarburos alifáticos (HA).

Al observar la (Tabla 1), y a modo de comparación con lo reportado para S. leucantha en la región norte de América y de Asia, es posible evidenciar similitudes en algunos compuestos determinados para la especie colectada en Colombia. Estos compuestos han sido reportados por Ali et al. (2014), quienes determinaron para S. leucanta, colectada en Winslow, Maine, Estados Unidos, la presencia de acetato de bornilo (11.4\%), y sesquiterpenos tales como cariofileno (6.5\%), óxido de cariofileno (13.5\%) y espatulenol (7.0\%). De igual forma, Upadhyaya et al. (2009) determinaron la composición química en el aceite esencial de hojas de S. leucantha colectada en la India, y reportaron el 57.6\% de sesquiterpenos dentro de la composición total del aceite, y específicamente la presencia de espatulenol (12.1\%), b-cariofileno (10.7\%), y a-himacaleno (10.5\%). En cuanto a monoterpenos, se encontró la presencia del acetato de bornilo (27.8\%), el cual fue el componente mayoritario para el aceite esencial. Con respecto a este último compuesto, autores como Negi et al. (2007), Rondon et al. (2005), y Rojas et al. (2010) revelaron valores correspondientes al porcentaje de área de $23.9 \%, 40.9 \%$ y $24.1 \%$, respectivamente para la misma especie en sus partes aéreas. Además de considerarse el componente mayoritario en la mezcla, cabe resaltar que reporta la presencia de algunos sesquiterpenos tales como cariofileno, germacreno D, y espatulenol en cuanto componentes representativos.

En comparación con lo reportado anteriormente, es posible resaltar que el aceite esencial extraído de flores de S. leucantha colectada en el municipio de Guavio, Colombia, presentó una diferencia significativa que corresponde a la cantidad en mayor medida de un sesquiterpeno tricíclico (aristoleno), con valores de porcentaje de área del 17.2\%. Para el monoterpeno acetato de bornilo fue del $9.8 \%$. Esta variabilidad respecto a las especies colectadas en otras partes puede deberse a que el presente trabajo se basó en la extracción específica del aceite esencial del órgano flor, motivo por el cual se destaca la importancia del trabajo concerniente a la separación de los órganos de la especie al momento de su análisis.

La comparación entre el aceite esencial extraído de S. leucantha en Colombia y lo reportado para el género Salvia permite corroborar la presencia de compuestos asociados a dicho género. Es en el caso de lo reportado por Ali et al. (2014), quienes reportan la composición química de cuatro especies de Salvia (S. apiana, S. elegans, S. officinalis y S. leucantha), es posible observar que en todas ellas se encuentran presentes los monoterpenos $\mathrm{a} y \mathrm{~b}$ pineno, además de d-canfeno. En cuanto a sesquiterpenos es posible corroborar la presencia en el género de algunos compuestos tales como g-terpineno, acetato de bornilo, b-elemeno, g-cadineno, cariofileno y óxido de cariofileno, entre otros, los cuales se podrían considerar como posibles marcadores quimiotaxonómicos en el género Salvia.

Adicionalmente, fue posible encontrar diferencias en cuanto a la composición porcentual de terpenos para S. leucantha respecto a otras especies del mismo género, de manera que para $S$. apiana se reporta un porcentaje del $77.3 \%$ de monoterpenos oxigenados, compuestos que para $S$. elegans y $S$. officinalis son del $40.3 \%$ y el $44.2 \%$, respectivamente 
(Ali et al., 2014), valor que para este estudio fue del $9.8 \%$. En cuanto a sesquiterpenos, fue posible observar que, para las especies reportadas por Ali et al. en el 2014, fueron reportados valores de 3.3\%, $7.4 \%$ y $2.8 \%$ para S. apiana, S. elegans y S. officinalis, mientras que en el presente estudio se reporta un valor del $58.8 \%$.

Algunas actividades biológicas han sido reportadas para el aceite esencial de S. leucantha. Tal es el caso de los resultados propuestos por Ali et al. (2014), quienes a partir del aceite esencial de la especie demostraron una fuerte actividad larvicida. De la misma manera, Upadhyaya et al. (2009) verificaron la actividad antioxidante para los compuestos presentes en el aceite esencial de la misma especie. Algunas otras actividades como la antifúngica y la antibacteriana reportadas por Kabouch y Kabouch (2008) han sido atribuidas específicamente para los sesquiterpenos presentes en la especie, lo que lleva a sugerir estudios con el aceite esencial de flores extraído de $S$. leucantha colectada en Colombia, encaminados a la evaluación de estas actividades biológicas de acuerdo con la composición química aquí reportada.

\section{Conclusiones}

Se determinó, por primera vez en Colombia, la composición química del aceite esencial de las flores de Salvia leucantha Cav, de modo que se estableció la presencia de sesquiterpenos en los que se encuentra el aristoleno como compuesto mayoritario con un porcentaje de 17.2\%. De igual forma, se reportaron algunos monoterpenos en los que se resalta la presencia de acetato de bornilo (9.8\%). Cabe destacar que muchos de los compuestos aquí determinados están de acuerdo con los reportados por otros autores, pero difieren en cuanto al compuesto mayoritario, el cual aquí corresponde a un sesquiterpeno, y para especies reportadas en otros continentes corresponde a un monoterpeno.

\section{Agradecimientos}

Los autores expresan sus agradecimientos a la Universidad Distrital Francisco José de caldas.

\section{Referencias}

[1] Adams, R. (2007). Identification Of Essential Oil Components By Gas Chromatography/Mass Spectroscopy. EE. UU.: Allured Publishing Corporation.

[2] Ali, A., Tabanca, N., Demirci, B., Blythe, E., Ali, Z., Husnu, C. y Khan, A. (2014). Chemical Composition and Biological Activity of Four Salvia Essential Oils and Individual Compounds against Two Species of Mosquitoes. Journal Of Agricultural And Food Chemistry, 63, 447-456.

[3] Aoyagi, Y., Yamazaki, A., Nakatsugawa, C., Fukaya, H., Takeya, K., Kawauchi, S. e Izumi, H. (2008). Salvileucalin B, a Novel Diterpenoid with an Unprecedented Rearranged Neoclerodane Skeleton from Salvia leucantha Cav. Organic letters, 10, 4429-4432.

[4] Amir, R., Somayeh, S., Omidreza, F., Jianbo y X. (2016). Bioactive Phytoquemicals from Shoots of Salvia Species. Phytochemistry Review, 15, 829-867.

[5] Cely, W., Matulevich, J. y Castrillon, W. (2014). Triterpenos y esteroles de Salvia leucantha (Lamiaceae) y evaluación de su capacidad antioxidante. Revista Facultad de Ciencias Básicas, 10, 68-79.

[6] Fernández, J. (2006). Revisión taxonómica de Salvia sect. Siphonantha (Labiatae). Anales del Jardín Botánico de Madrid, 63, 145-157.

[7] Goodner, K. (2008). Practical Retention Index Models Of OV-101, DB-1, DB-5, And DB-Wax For Flavor And Fragrance Compounds. LWT-Food Science and Techno$\log y, 41,951-958$.

[8] Jassbi, A., Zare, S., Firuzi, O. y Xiao, J. (2016). Bioactive Phytochemicals From Shoots And Roots Of Salvia Species. Phytochemistry Review, 15, 829-867.

[9] Kabouch, A. y Kabouch, Z. (2008). Bioactive Diterpenoids of Salvia Species. Studies in Natural Products Chemistry, 35, 753-832.

[10] Lia, L., Qia, Q., Liuc, S., Wua, X. y Zhaoa, Q. (2018). Neo-Clerodane and Abietane Diterpenoids With Neurotrophic Activities from the Aerial Parts of Salvia Leucantha Cav. Fitoterapia, 127, 367-374.

[11] Negi, A., Mohammad, S., Javed, A. y Anand, B. (2007). Steam Volatile Terpenoids from Salvia Leucantha. Journal of Essent Oil Research, 19, 463-465.

[12] Rojas, L., Visbal, T., Morillo, M., Rojas, Y., Arzola, J. y Usubillaga, A. (2010). The Volatile Constituents Of Salvia Leucantha. Natural Products Communications, 6, 937-938.

[13] Rondon, M., Velásco, J., Morales, A., Rojas, J., Carmona, J., Gualtieri, M. y Hernández, V. (2005). Composi- 
tion and Antibacterial Activity of The Essential Oil of Salvia Leucantha Cav. cultivated in Venezuela Andes. Revista Latinoamericana de Química, 33(2), 55-59.

[14] Upadhyaya, K., Dixit, V., Padalia, R. y Mathela, C. (2009) Terpenoid Composition and Antioxidant Activity of Essential Oil from Leaves o Salvia Leucantha Cav. Journal of Essential Oil Bearing Plants, 12, 551556. 
